\title{
Measurement Uncertainty Evaluation Method Considering Correlation and its Application to Precision Centrifuge
}

\author{
Mingxiang Ling, Huimin Li, Qisheng Li \\ Institute of System Engineering, CAEP, Mianshan Street, 32, Sichuan, China, ling_mx@163.com
}

\begin{abstract}
Measurement uncertainty evaluation based on the Monte Carlo method (MCM) with the assumption that all uncertainty sources are independent is common. For some measure problems, however, the correlation between input quantities is of great importance and even essential. The purpose of this paper is to provide an uncertainty evaluation method based on MCM that can handle correlated cases, especially for measurement in which uncertainty sources are correlated and submit to non-Gaussian distribution. In this method, a linear-nonlinear transformation technique was developed to generate correlated random variables sampling sequences with target prescribed marginal probability distribution and correlation coefficients. Measurement of the arm stretch of a precision centrifuge of $10^{-6}$ order was implemented by a high precision approach and associated uncertainty evaluation was carried out using the mentioned method and the method proposed in the Guide to the Expression of Uncertainty in Measurement (GUM). The obtained results were compared and discussed at last.
\end{abstract}

Keywords: Measurement uncertainty, Monte Carlo method, correlation, precision centrifuge, correlated random sampling.

\section{INTRODUCTION}

$\mathrm{M}$ EASUREMENT uncertainty is one of the most important parameters for lab metering, geometrical product specification and quality assurance, especially when the measurement result is part of a measurement chain tracing back to national standards. So it is necessary to give the uncertainty associated with measurement.

GUM is an internationally accepted standard document for uncertainty evaluation, which is based on the law of propagation of uncertainty (LPU) [1]. In GUM, correlation between input quantities of measurand is easy to handle, and for the cases where the mathematical model of measurand is strongly non-linear, it would also be helpful to have recourse to LPU with the second or higher-order Taylor series expansion [2]. However, for cases where computation of partial derivatives is difficult or the output quantity is not characterized by a Gaussian distribution nor a scaled and shifted $t$-distribution, the GUM may be inadequate, whereas the supplement 1 to GUM-Propagation of Distributions using a Monte Carlo method (GUM S1) [3] is expected to perform well in such cases and has been recommended.

In GUM S1, propagation of uncertainty of input quantities to measurand is based on probability density functions (PDFs) by using the Monte Carlo simulation. Since GUM $\mathrm{S} 1$ can provide guidance when the conditions for GUM are unclear or are not fulfilled, MCM has been employed in evaluating uncertainty by many researchers recently [4-10]. So far, uncertainty evaluation based on MCM is studied widely and some researchers also consider correlation in their uncertainty calculation [11]. The fact is still that, however, MCM-based measurement uncertainty evaluation method considering correlation between input quantities is limited. For some measurement problems, considering correlation is important and even essential. For example, when input parameters of measurand are derived from the same instrument, the same reference or are physically correlated, one should seriously confirm if these data can be treated as independent. For these complicated measurement problems, GUM S1 recommends theoretically sampling from the joint PDF of input quantities; still it is very difficult to implement in practice. So a few researchers focused mainly on considering correlation of input quantities with Gaussian distribution [3, 12-14], and there is limited information available in literature about MCM-based uncertainty evaluation methods with correlated but nonGaussian distributed input quantities. In fact, uncertainty sources deriving from instrument or reference are more often treated as Uniform or Triangular distribution but not Gaussian distribution [15]. Therefore, it is necessary to develop a MCM-based uncertainty evaluation method that can handle measurement problems with correlated and any probability density distributed input quantities.

Based on GUM S1, we extended uncertainty evaluation to measurement problems with correlated input quantities, the principle and process is given in Section 2, and a generalized method for generating correlated random sampling sequences with target PDF is developed in Section 3. Arm stretch of a $10^{-6}$ precision centrifuge is measured and associated uncertainty is evaluated in Section 4. At last, conclusion remarks are made in Section 5.

\section{EVALUATION METHOD CONSIDERING CORRELATION}

In a Bayesian approach to measurement uncertainty evaluation, the measurement result $y$ of measurand $Y$ is the expectation of the measurand

$$
y=\int g_{Y}(\eta) \eta d \eta
$$

Probability density function $g_{Y}(\eta)$ encodes the given information about $Y$ and $\eta$ denotes a possible value that can be attributed to $Y$. The standard uncertainty $u(y)$ associated with the measurement result is the standard deviation of $y$

$$
u^{2}(y)=\int g_{Y}(\eta)(\eta-y)^{2} d \eta
$$


When measurand $Y$ depends on several input quantities $X_{1}$, $\ldots, X_{N}$, for which information is available by a joint PDF $g_{X 1}, \ldots, X N\left(\xi_{1}, \ldots, \xi_{N}\right)$ and the possible values of the input quantities are denoted as $\left(\xi_{1}, \ldots, \xi_{N}\right)$, it is propagated through the model function $Y=f\left(X_{1}, \ldots, X_{N}\right)$ to yield the PDF $g_{Y}(\eta)$ associated with the measurand $Y$. Consequently, the best estimate $y$ of $Y$ and associated standard uncertainty $u(y)$ can be stated as [16]

$$
\begin{gathered}
y=\int_{-\infty}^{+\infty} \cdots \int_{-\infty}^{+\infty} g_{X_{1}, \ldots, X_{N}}\left(\xi_{1}, \ldots, \xi_{N}\right) f\left(\xi_{1}, \ldots, \xi_{N}\right) d \xi_{1} \cdots d \xi_{N} \\
u^{2}(y)=\int_{-\infty}^{+\infty} \cdots \int_{-\infty}^{+\infty} g_{X_{1}, \ldots, X_{N}}\left(\xi_{1}, \ldots, \xi_{N}\right)\left[f\left(\xi_{1}, \ldots, \xi_{N}\right)-y\right]^{2} d \xi_{1} \cdots d \xi_{N}
\end{gathered}
$$

MCM provides a practical and generally applicable tool for calculating the multidimensional integrals (3) and (4), especially for a large number of input quantities and complex model function $f\left(X_{1}, \ldots, X_{N}\right)$. In the Monte Carlo simulation, a random selection of combinations of possible values of input quantities is made to propagate the distributions associated with these quantities. And for independent or typical correlated input quantities (e.g., joint Gaussian distribution), MCM is implemented easily by drawing random deviates from the distribution $g_{X i}(\xi), i=1$, $\ldots, N$, and propagating these deviates through model function of measurand to yield random deviates distributed as $g_{Y}(\eta)$. Repeating this procedure many times, an empirical distribution of $g_{Y}(\eta)$ is obtained, from which the estimate and associated standard uncertainty of $Y$ as well as coverage intervals can be determined.

For complex measurement problems with correlated and non-Normal distributed input quantities, however, the situation is more troublesome, and the above Monte Carlo procedure recommended by GUM S1 [3] is unfitted, for it is very hard to assign a joint PDF $g_{X 1, \ldots, X N}\left(\xi_{1}, \ldots, \xi_{N}\right)$ for input quantities in practice. Usually, the prescribed marginal probability distribution and correlation coefficients of input quantities are available by using previous information from manufacture's certificates or analyst's experience. Consequently, drawing random deviates from the correlated joint PDF $g_{X 1}, \ldots,{ }_{X N}\left(\xi_{1}, \ldots, \xi_{N}\right)$ can be carried out by employing a linear-nonlinear technique. The corresponding Monte Carlo simulation steps, shown in Fig.1. as a schematic flow graph, are as follows: Independent random sequences uniformly distributed in the interval $[0,1]$ are generated, dimensionality of the sequences equals to the number of input quantities and the length of each dimension is the Monte Carlo trial times $M$; Then, the independent random sequences in the interval $[0,1]$ are converted according to the PDFs and correlation coefficients of input quantities to independent random sequences, correlated Gaussian distributed sequences or correlated non-Gaussian distributed sequences, respectively, by adopting three methods (illustrated in Fig.1.); Last, the mean and associated uncertainty of output quantities as well as coverage interval at a given coverage level are calculated.

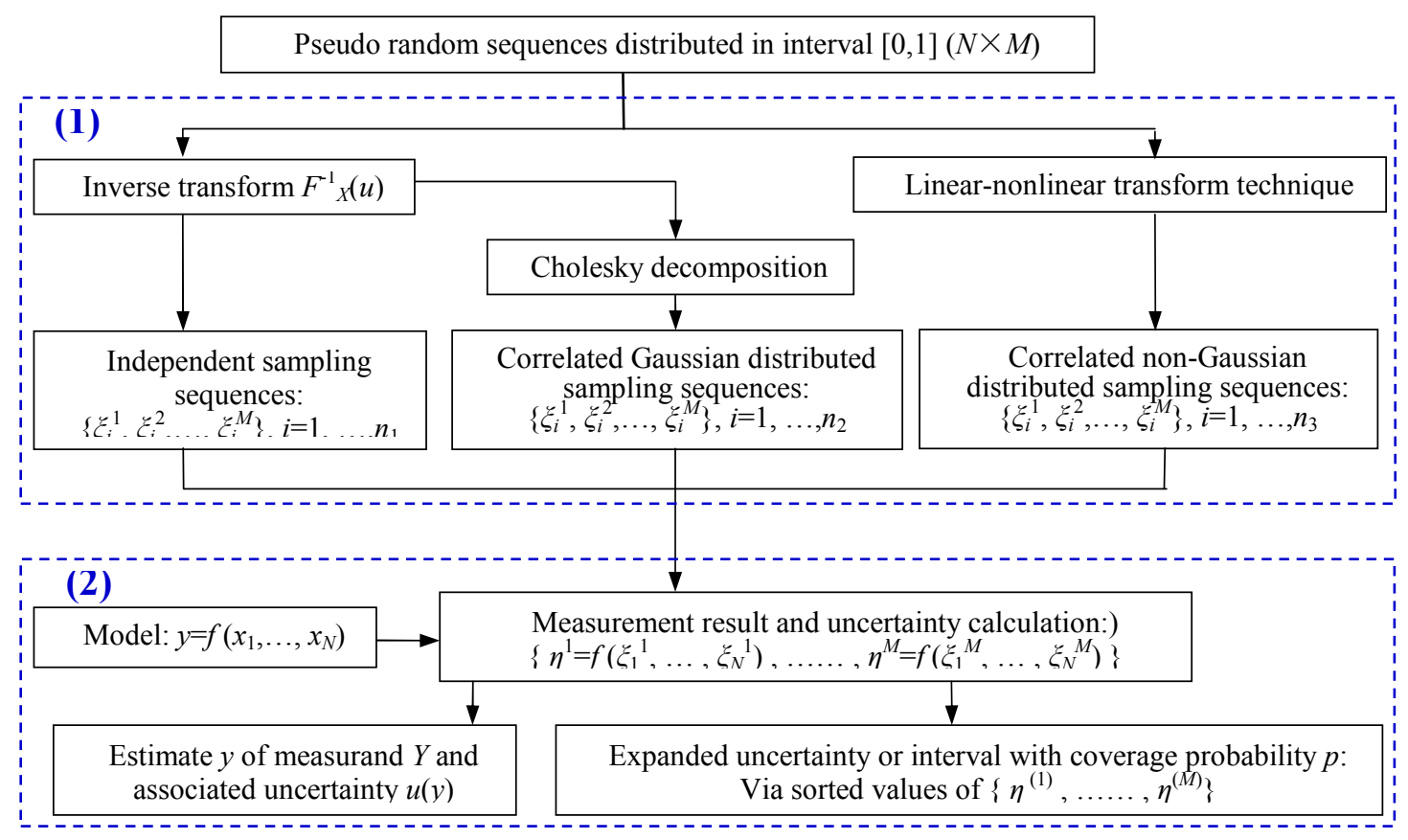

Fig.1. Schematic flow graph of uncertainty evaluation based on MCM considering correlation. 


\section{A. Sampling from the PDFs of input quantities.}

We classify input quantities of measurand as independent quantities (denotes by $X_{1}, X_{2}, \ldots, X_{n 1}$ ), correlated quantities with Gaussian distribution (denotes by $X_{n 1+1}, X_{n 1+2}, \ldots$, $\left.X_{n 1+n 2}\right)$ and correlated quantities with non-Gaussian distribution (denotes by $X_{n 1+n 2+1}, X_{n 1+n 2+2}, \ldots, X_{n 1+n 2+n 3}$ ), apparently ' $n_{1}+n_{2}+n_{3}$ 'equal to the number of input quantities $N$. Moreover, the three types do not necessarily exist simultaneously in a measurement problem.

The sampling values of independent input quantities $X_{1}$, $X_{2}, \ldots, X_{n 1}$, denoted as $\left(\xi_{1}, \ldots, \xi_{n 1}\right)$, are calculated by means of inversion of corresponding cumulative distribution functions (CDFs) of each input quantity

$$
\xi_{1}=F_{X_{1}}^{-1}\left(u_{1}\right), \quad \xi_{2}=F_{X_{2}}^{-1}\left(u_{2}\right), \quad \cdots, \quad \xi_{X_{n 1}}=F_{X_{n 1}}^{-1}\left(u_{X_{n 1}}\right)
$$

Where $u_{r}, r=1,2, \ldots, n_{1}$, is pseudo random sequences (vector), and $F_{X r}^{-1}($.$) is the CDF of input quantity X_{r}$.

For sampling values of mutually correlated input quantities $X_{n 1+1}, X_{n 1+2}, \ldots, X_{N}$, the generating procedure is illustrated in detail in Section 3.

\section{B. Output quantity and associated uncertainty calculation.}

When sampling values of input quantities are generated, the next step is calculating $\left\{\eta^{j}=f\left(\xi_{1}{ }^{i}, \ldots, \xi_{N}{ }^{i}\right)\right\}, i=1, \ldots, M$, by using model function $Y=f\left(X_{1}, \ldots, X_{N}\right)$. The series $\left\{\eta^{1}, \eta\right.$ $\left.{ }^{2}, \ldots, \xi^{M}\right\}$ constitute a set of independent random deviates from the PDF of $Y$. Then, calculate the mean and standard deviation of $\left\{\eta^{1}, \eta^{2}, \ldots, \xi^{M}\right\}$ as the measurement result $y$ and associated standard uncertainty $u(y)$ for measurand $Y$. Lastly, sort the values $\left\{\eta^{1}, \eta^{2}, \ldots, \xi^{M}\right\}$ in ascending order, $\eta^{(1)} \leq \eta^{(2)} \leq \ldots \leq \xi^{(M)}$, and determine a shortest coverage interval $\left[\eta^{(L)} \leq \eta^{(H)}\right]$ at coverage probability $p$ where $H$ - $L$ equals to the integer part of $p \cdot M+1 / 2$.

As $M \rightarrow+\infty$, these calculation results converge to the optimal value of the measurand. For finite $M$, however, the results of MCM contain a random error, and $M$ must therefore be chosen sufficiently large (e.g., $\geq 10^{6}$ ) to ensure a small calculation error, corresponding guidance for choosing an appropriate $M$ can be seen in $[3,17]$.

\section{SAMPLING SEQUENCES OF CORRELATED INPUT QUANTITIES GENERATING}

The goal of this section is to generate a $n \times M$-dimensional random sampling sequence $\boldsymbol{X}=\left(x_{1 j}, \ldots, x_{n j}\right)^{\mathrm{T}}$ with the target marginal distribution function $F_{X k}(\xi)$ and correlation coefficient matrix $R_{x}$, here $n$ is the number of correlated input quantities of measurand, $j=1,2, \ldots, M, k=1,2, \ldots, n$. An appropriate methodology for this goal is based on linearnonlinear transformation detailed by Li and Hammond [18], Marida [19] and Cairo [20], et al. In this algorithm, random sampling sequences with target marginal probability distribution can be obtained by transforming non-linearly elements of a sample from a multivariate standard Normal distribution (corresponding random vector is denoted as $Z$ ) with correlation coefficients matrix $R_{Z}$, and the sampling values of the correlated multivariate standard Normal distribution are transformed linearly from different sampling sequences (denoted as vector $\boldsymbol{V}$ ) of independent standard Normal distribution by the Cholesky factor. The whole procedure is illustrated in Fig.2., and can be viewed as a three-step process. In the first step, correlation matrix $R_{Z}$ is calculated by the desired correlation matrix $R_{X}$. The second step involves linearly transforming an independent standard Normal distributed variable $\boldsymbol{V}$ into a correlated multivariate Normal distributed variable $\boldsymbol{Z}$. In the last step, the target distributed variable $\boldsymbol{X}$ is transformed non-linearly from $\boldsymbol{Z}$.

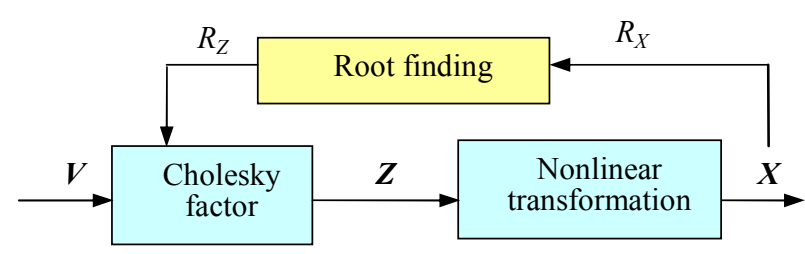

Fig.2. Procedure of generating correlated random sampling sequences with target probability distribution.

According to the principle of cumulative probability distribution equivalency, sequences $\boldsymbol{X}$ can be calculated by

$$
\boldsymbol{X}=\boldsymbol{H}(\boldsymbol{Z})=\left(F_{x_{1}}^{-1}\left[\phi\left(\boldsymbol{z}_{1}\right)\right], F_{x_{2}}^{-1}\left[\phi\left(\boldsymbol{z}_{2}\right)\right], \mathrm{L}, F_{x n}^{-1}\left[\phi\left(\boldsymbol{z}_{n}\right)\right]\right)^{T}
$$

where vector $\boldsymbol{z}_{k}=\left(z_{k 1}, z_{k 2}, \ldots, z_{k M}\right)^{\mathrm{T}}, k=1,2, \ldots, n$, denotes sampling sequences from $n$-dimensional joint Normal distributed variable $Z$. $F^{-1}{ }_{X}$ is the inverse CDF of $X$ and $\Phi$ is the standard Normal cumulative probability function

$$
\phi(z)=\frac{1}{2}\left(1+\operatorname{erf}\left(\frac{z}{\sqrt{2}}\right)\right)=\frac{1}{2}+\frac{1}{\sqrt{\pi}} \int_{0}^{z / \sqrt{2}} \exp \left(-t^{2}\right) d t
$$

The transformation function $F_{X k}^{-1}[\Phi()$.$] ensures that X_{k}$ has the target marginal distribution $F_{X k}$. Therefore, the important problem is to calculate the correlation coefficient matrix $R_{Z}$ that is used to generate correlated Normal sampling sequences, then the correlated sampling sequences of target random variable $X$ can be generated by (6). Here, the correlation coefficient matrices $R_{X}$ and $R_{Z}$ are stated as

$$
R_{X}=\left[\begin{array}{cccc}
1 & r_{x_{1} x_{2}} & \cdots & r_{x_{1} x_{n}} \\
r_{x_{1} x_{2}} & 1 & \cdots & r_{x_{2} x_{n}} \\
\vdots & \vdots & \cdots & \vdots \\
r_{x_{1} x_{n}} & r_{x_{2} x_{n}} & \cdots & 1
\end{array}\right], R_{Z}=\left[\begin{array}{cccc}
1 & r_{z_{1} z_{2}} & \cdots & r_{z_{1} z_{n}} \\
r_{z_{1} z_{2}} & 1 & \cdots & r_{z_{2} z_{n}} \\
\vdots & \vdots & \cdots & \vdots \\
r_{z_{1} z_{n}} & r_{z_{2} z_{n}} & \cdots & 1
\end{array}\right]
$$

According to the definition of correlation coefficient, the mathematical relationship between $R_{Z}$ and $R_{X}$ can be eventually expressed as [18]

$$
\begin{aligned}
r_{x_{i} x_{j}}= & \frac{E\left[\left(x_{i}-\mu_{i}\right)\left(x_{j}-\mu_{j}\right)\right]}{\sigma_{i} \sigma_{j}}=\int_{-\infty}^{+\infty} \int_{-\infty}^{+\infty} \frac{\left(H\left(z_{i}\right)-\mu_{i}\right)\left(H\left(z_{j}\right)-\mu_{j}\right)}{\sigma_{i} \sigma_{j}} \\
& \cdot \exp \left(-\frac{z_{i}^{2}-2 r_{z_{i} z_{j}} z_{i} z_{j}+z_{j}^{2}}{2\left(1-r_{z_{i} z_{j}}^{2}\right)}\right) \frac{1}{2 \pi \sqrt{1-r_{z_{i} z_{j}}^{2}}} d z_{i} d z_{j} \quad i \neq j
\end{aligned}
$$


where, $\mu_{i}, \sigma_{i}$ is the expectation and standard deviation of $x_{i}$. For many marginal distributions, (9) is very complicated and is usually unsolvable by analytic methods and must be in virtue of numerical calculation. Cairo [20] and Hammond [18] employed Newton's method and Raphson Quadrature formula to solve (9), while Yen [21] applied Gaussian Quadrature integration and Seyed [22] used artificial neural networks. In these methods, besides the complexity of algorithm, the computational times also increase quadratically with $n$ and may be inaccurate for ill-suited initial values. To avoid the problems involved in solving (9), here we build an objective function and used optimal search strategy to calculate the values of $r_{z i z j}$ corresponding to the target values of $r_{x i x j}$. The objective function can be selected as

$$
\text { Search } \quad r_{z_{i} z_{j}}, \quad \text { Let } \min \left\{\left|r_{x_{i} x_{j}}-\int_{-\infty}^{+\infty} \int_{-\infty}^{+\infty} f\left(z_{i}, z_{j}, r_{z_{i} z_{j}}\right) d z_{i} d z_{j}\right|\right\}
$$

where

$$
f\left(z_{i}, z_{j}, r_{z_{i} z_{j}}\right)=\frac{\left(H\left(z_{i}\right)-\mu_{i}\right)\left(H\left(z_{j}\right)-\mu_{j}\right)}{\sigma_{i} \sigma_{j}}
$$

$$
\cdot \exp \left(-\frac{z_{i}^{2}-2 r_{z_{i} z_{j}} z_{i} z_{j}+z_{j}^{2}}{2\left(1-r_{z_{i} z_{j}}^{2}\right)}\right) \frac{1}{2 \pi \sqrt{1-r_{z_{i} z_{j}}^{2}}}
$$

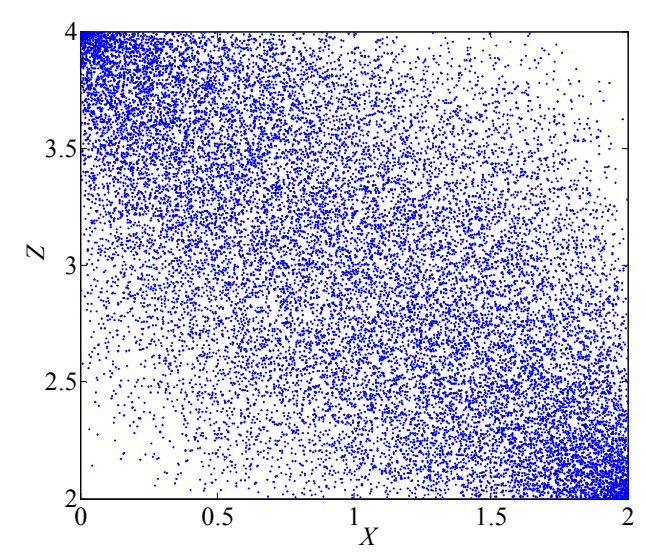

a) The trend of statistical values of $X$ and $Z$.

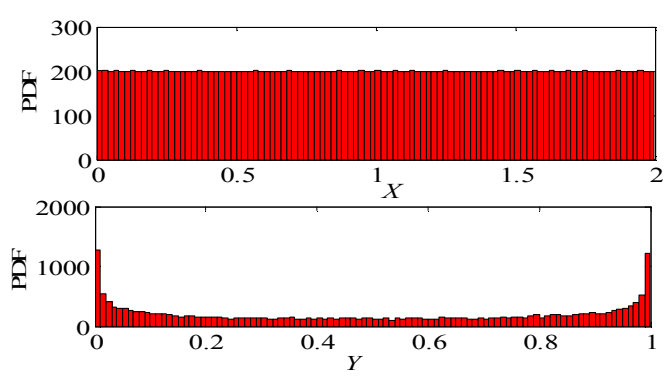

b) Histograms of random variables $X$ and $Y$.

Fig.3. Sampling sequences distribution with 20,000 points and histograms of random variables $X, Y$ and $Z$. In Fig.3.a), the trend of statistical values of $X$ and $Z$ is inverse as the corresponding correlation coefficient is subtractive. Fig.3.b) shows the empirical frequency distributions of random variables $X$ and $Y$, which clearly illustrates the high consistency of sampling sequences' statistical PDF with the given theoretical probability distribution.
Genetic Algorithm is employed to solve (10), which has been proved to be an efficient search strategy for nonlinear root finding problems and there is no need to set appropriate initial values. Besides, the up and down limit of integral within interval $[-4,4]$ is accurate enough for bivariate Normal distribution in (10).

To verify the proposed method, three random sampling sequences with uniform distributed variable $X$ in the interval $[0,2]$, arcsine distributed variable $Y$ in the interval $[0,1]$, and uniform distributed variable $Z$ in the interval $[2,4]$, for which the target correlation coefficient matrix is given in (11), are generated by the proposed method. The distribution relationships between the three sampling sequences with 20,000 points are illustrated in Fig.3. Actual correlation coefficients of the sampling sequences are also calculated and the results are shown in (11).

As can be seen from Fig.3. and (11), random sampling sequences generated by the proposed method approximately submit to the target probability distribution and the desired correlation coefficients.

$$
r_{x_{i} x_{j}}=\left[\begin{array}{ccc}
1.0 & 0.5 & -0.6 \\
0.5 & 1.0 & 0.0 \\
-0.6 & 0.0 & 1.0
\end{array}\right] \%_{x_{i} x_{j}}^{\sigma_{j}}=\left[\begin{array}{ccc}
1.0000 & 0.5012 & -0.5976 \\
0.5012 & 1.0000 & 0.0007 \\
-0.5976 & 0.0007 & 1.0000
\end{array}\right]
$$

\section{MEASUREMENT UNCERTAINTY EVALUATION FOR ARM STRETCH OF PRECISION CENTRIFUGE}

Precision centrifuge is a motor driven arm structure device for calibrating the performance parameters of inertial accelerometer or other inertial instruments. For high precision centrifuge, whose relative standard uncertainty of output acceleration reaches to $10^{-6}$ order, the measurement and associated uncertainty evaluation of dynamic changes in radius due to centripetal loading, dynamic center of rotation, and thermal expansion is essential [23], thus, there is a significant contribution of measurement uncertainty to output acceleration, which further affects calibration accuracy of inertial accelerometer. Micron, even submicron measurement uncertainty can be valuable for high precision calibrating tests and the influence of correlation between uncertainty sources derived from senses, mechanical installation errors, and data acquisition system is enormous and must be considered.

A practical precision centrifuge's arm stretch was measured (Fig.4.). The precision centrifuge can provide an acceleration benchmark at the range of $1 \mathrm{~g}$ to $100 \mathrm{~g}$ with the relative standard uncertainty of $10^{-6}$, which is used to calibrate model parameters of high precision accelerometer in inertial navigation.

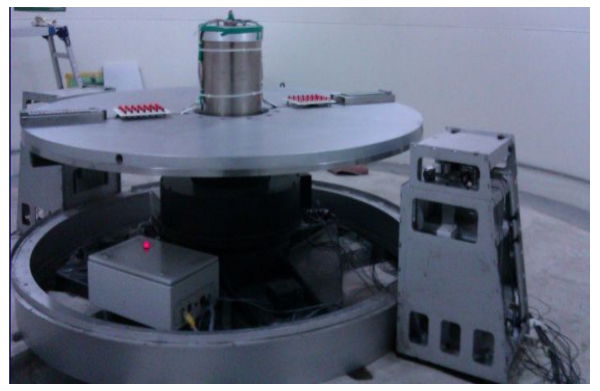

Fig.4. Picture of precision centrifuge with the acceleration relative standard uncertainty of $10^{-6}$. 


\section{A. Measurement principle of arm stretch.}

The measurement for arm stretch of precision centrifuge is based on capacitance micrometer (Fig.5.). The capacitance micrometer is fixed in a precision clamp and fine-tuned to ensure the sense is aligned to the edge of positioning stage mounted on the centrifuge at an initial distance of about $400 \mu \mathrm{m}$. Here the positioning stage is used to fix the tested inertial accelerometer.

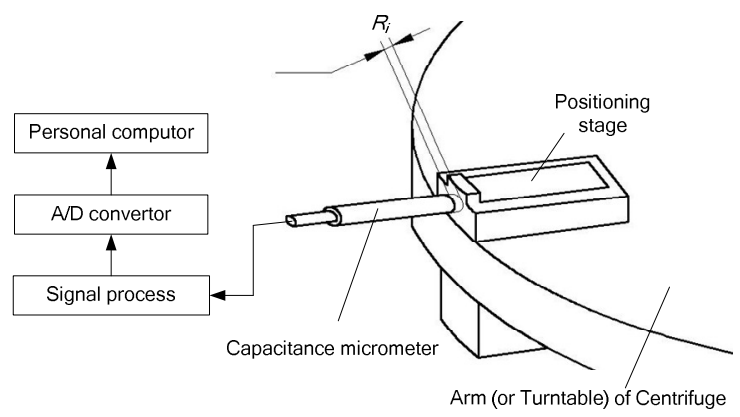

Fig.5. Schematic diagram of measuring the arm stretch of precision centrifuge. $R_{i}, i=1,2$, is the direct space between sensor of the capacitance micrometer and the edge of positioning stage when centrifuge is in static state and work state, respectively.

When precision centrifuge circumgyrates at target rotational angular velocity of $\omega$, the turntable(radius) will be stretched, and as the positioning stage arrives at the location of capacitance micrometer, output of the capacitance micrometer will turn into effective values from over-range state. Direct distance between the sensor of capacitance micrometer to the edge of positioning stage under the static state and test state with target $\omega$ can be measured. And then the data output by capacitance micrometer is calculated on a PC after being sampled by an A/D converter. The arm stretch $\Delta R$ equals to the difference by subtracting direct distance between the sensor of capacitance micrometer to the edge of positioning stage at target $\omega$ from the corresponding distance when the centrifuge is static.

\section{B. Modeling and identification of uncertainty sources.}

According to the above principle, the mathematical model of measuring $\Delta R$ can be expressed as

$$
\Delta R=R_{1}-R_{2}
$$

Where, $R_{1}$ is direct distance between the sensor of capacitance micrometer and the edge of positioning stage when the centrifuge is in static state, while $R_{2}$ corresponds to the test state at a target rotational angular velocity of $\omega$.

The following significant sources of uncertainty that affect the measurand $\Delta R$ can be identified:

1. Repeatability of $R_{1}$ and $R_{2}$. To reduce the influence of electrical noises or other random errors, the mean of repeated readings of output values in the same environment condition was adopted as the measurement result. And the corresponding uncertainty can be estimated as a type A component, which can be classified as deriving from random effects.

2. Indicating error of the capacitance micrometer due to nonlinearity, hysteresis and thermal drift of sensor.
3. Mechanical installation error of capacitance micrometer. Ideally, the central axis of capacitance micrometer must be vertical to the edge of positioning stage, whereas there is a misalignment error in practice.

4. Error of $\mathrm{A} / \mathrm{D}$ convertor due to thermal drift, zero drift, plus error of data acquisition.

5. Roundness error of the positioning stage. There is a residual roundness error contained in the output values of capacitance micrometer. Although the positioning stage is processed mechanically with ultra-smooth surface, a point to point trigger measurement strategy is still used to reduce the roundness error.

Usually, the error sources mentioned in b) e) must be compensated for $\Delta R$ and associated uncertainty must be considered into the total measurement uncertainty of $\Delta R$. These uncertainty sources are usually available by using previous information from certificates or analyst's experience, and can be estimated as a type B component, which can be classified as deriving from systematic effects. Considering the above error sources, $\Delta R$ can be modified as

$$
\Delta R=\left(\frac{1}{q} \sum_{k=1}^{q} R_{1 k}+\alpha_{1}+\beta_{1}+\gamma_{1}+\delta_{1}\right)-\left(\frac{1}{q} \sum_{k=1}^{q} R_{2 k}+\alpha_{2}+\beta_{2}+\gamma_{2}+\delta_{2}\right)
$$

where, parameters of all input components are presented in Table 1 . As an uncertainty budget, it lists the most important contributors to measurement uncertainty of $\Delta R$ and takes into account all their necessary parameters.

Defined parameters and associated uncertainty components are listed in Table 1. The data in the first and second row were quantified from experimental work, and associated uncertainty was regarded as Type A component deriving from random effects. During the process of measurement, the temperature was controlled within $20 \pm 0.5^{\circ} \mathrm{C}$ and grounded resistance was controlled within $50 \mathrm{~m} \Omega$ in our laboratory. Detailed Mean values of $R_{2}$ and associated standard deviation at different rotational angular velocities are listed in Table 2. Because the uncertainty components derived from repeatability at all rotational angular velocities are larger than the uncertainty components from resolution of instruments, the latter is not budgeted in Table 1.

For other type B components in Table 1, all we can confirm is the confidence interval, the defined values for these components are acquired from verification certificates or experimental judgement. Estimates of these components are recognized as zero, but the associated uncertainty must be calculated and propagated. Uniform distributions are assigned for capacitance micrometer's indication error $\alpha_{1}, \alpha_{2}$ and $\mathrm{A} / \mathrm{D}$ converter error $\beta_{1}, \beta_{2}$, which is common treatment in GUM. For installation error $\gamma_{1}$ and $\gamma_{2}$, ideal state is leaving the axis of the capacitance micrometer vertical to the plane of measurement. Unfortunately, there is always an error brought by installation of capacitance micrometer, and we can confirm that installation error is not possible to beyond a certain value in view of phenomenological prediction. Through fixing capacitance micrometer in a precision fixture and fine-tuning repeatedly, installation error tends to be close to zero, so the probability frequency distribution can be regarded as maximum near zero and becomes smaller for larger installation errors. Based on the above analysis, Triangular distribution is assigned for $\gamma_{1}$ and $\gamma_{2}$ 
Table 1. Measurement results and associated uncertainty budget for arm stretch.

\begin{tabular}{|c|c|c|c|c|c|c|c|}
\hline Symbol & Description & $\begin{array}{l}\text { Estimation } \\
/ \mu \mathrm{m}\end{array}$ & Distribution & Division & $\begin{array}{c}\text { Standard } \\
\text { uncertainty } \\
/ \mu \mathrm{m}\end{array}$ & Type & Correlation \\
\hline$R_{1}$ & Mean value of $R_{1}$ & 430.69 & Normal & 1 & 0.019 & A & Independent \\
\hline$R_{2}$ & Mean value of $R_{2}$ & See Table 2 & Normal & 1 & See Table 2 & $\mathrm{~A}$ & Independent \\
\hline$\alpha_{1}$ & $\begin{array}{l}\text { Corrected value of capacitance } \\
\text { micrometer's indication error }\end{array}$ & 0 & Uniform & $\sqrt{3}$ & 0.121 & B & \multirow{2}{*}{$\begin{array}{l}\text { Mutually } \\
\text { correlated }\end{array}$} \\
\hline$\alpha_{2}$ & $\begin{array}{l}\text { Corrected value of capacitance } \\
\text { micrometer's indication error }\end{array}$ & 0 & Uniform & $\sqrt{3}$ & 0.121 & B & \\
\hline$\beta_{1}$ & $\begin{array}{l}\text { Corrected value of A/D converter } \\
\text { error }\end{array}$ & 0 & Uniform & $\sqrt{3}$ & 0.058 & B & \multirow{2}{*}{$\begin{array}{l}\text { Mutually } \\
\text { correlated }\end{array}$} \\
\hline$\beta_{2}$ & $\begin{array}{c}\text { Corrected value of } \mathrm{A} / \mathrm{D} \text { converter } \\
\text { error }\end{array}$ & 0 & Uniform & $\sqrt{3}$ & 0.058 & B & \\
\hline$\gamma_{1}$ & $\begin{array}{l}\text { Corrected value of installation error } \\
\text { of capacitance micrometer }\end{array}$ & 0 & Triangular & $\sqrt{6}$ & 0.102 & B & \multirow{2}{*}{$\begin{array}{l}\text { Mutually } \\
\text { correlated }\end{array}$} \\
\hline$\gamma_{2}$ & $\begin{array}{l}\text { Corrected value of installation error } \\
\text { of capacitance micrometer }\end{array}$ & 0 & Triangular & $\sqrt{6}$ & 0.102 & B & \\
\hline$\delta_{1}$ & $\begin{array}{l}\text { Corrected value of roughness error of } \\
\text { positioning stage }\end{array}$ & 0 & Normal & 1 & 0.018 & A & Independent \\
\hline$\delta_{2}$ & $\begin{array}{l}\text { Corrected value of roughness error of } \\
\text { positioning stage }\end{array}$ & 0 & Normal & 1 & 0.018 & A & Independent \\
\hline
\end{tabular}

Table 2. Mean values and standard deviation of $R_{1}$ and $R_{2}$ at different rotational angular velocities. At each rotational angular velocity, the trial times of repeated measurement are 20 .

\begin{tabular}{|c|c|c|c|}
\hline Symbol & $\begin{array}{c}\boldsymbol{\omega}=\mathbf{5 . 2 4} \\
\mathbf{r a d} / \mathbf{s}\end{array}$ & $\begin{array}{c}\boldsymbol{\omega}=\mathbf{2 0 . 9 6} \\
\mathbf{r a d} / \mathbf{s}\end{array}$ & $\begin{array}{c}\boldsymbol{\omega}=\mathbf{3 1 . 4 2} \\
\mathbf{r a d} / \mathbf{s}\end{array}$ \\
\hline Mean value of $R_{2} / \mu \mathrm{m}$ & 429.33 & 425.14 & 421.58 \\
\hline $\begin{array}{c}\text { Standard deviation of } \\
R_{2} / \mu \mathrm{m}\end{array}$ & 0.0471 & 0.0529 & 0.0426 \\
\hline
\end{tabular}

Correlation coefficient between $\gamma_{1}$ and $\gamma_{2}$ is recognized as strong, since mechanical installation error of capacitance micrometer affects coequally the output values $R_{1}$ and $R_{2}$. As indication spectrum of capacitance micrometer and A/D convertor are in the same range when measuring $R_{1}$ and $R_{2}$, which can be seen from Table 1. and Table 2., correlation coefficients between $\alpha_{1}$ and $\alpha_{2}, \beta_{1}$ and $\beta_{2}$ can be also expected to strengthen. In order to identify quantitatively these correlation coefficients, mean values of $R_{1}$ and $R_{2}$, which is sensed by capacitance micrometer and then output by $\mathrm{A} / \mathrm{D}$ convertor, are processed according to the following formula with repeated measurement trial times of 20

$$
r=\frac{\sum_{i=1}^{q}\left(R_{1 i}-\bar{R}_{1}\right)\left(R_{2 i}-\bar{R}_{2}\right)}{q(q-1)}
$$

where, $r$ denotes correlation coefficient, $q$ denotes the number of independent measurements, $\bar{R}_{1}, \bar{R}_{2}$ denote the mean values of arm stretch, corresponding results at different rotational angular velocity are presented in Table 1. and Table 2., and correlation coefficients are about 0.77 and 0.73 , respectively, when target $\omega$ is $5.24 \mathrm{rad} / \mathrm{s}$ and 20.96 . For the sake of simplicity, correlation coefficients of $\alpha_{1}$ and $\alpha_{2}, \beta_{1}$ and $\beta_{2}, \gamma_{1}$ and $\gamma_{2}$ are all assigned as 0.8 , which will bring a very small deviation with other adjacent values.

\section{Uncertainty evaluation by two methods and discussion.}

Measurement model (13) is linear with correlated input quantities, so the uncertainty propagation model with the first-order Taylor series expansion is accurate enough for measurand $\Delta R$ based on the GUM uncertainty framework. Here, we give the mathematical model for estimation of the standard uncertainty of $\Delta R$, considering the correlation between input components

$$
u(\Delta R)=\sqrt{\begin{array}{l}
u^{2}\left(R_{1}\right)+u^{2}\left(R_{2}\right)+u^{2}\left(\alpha_{1}\right)+u^{2}\left(\alpha_{2}\right)-2 r_{1} u\left(\alpha_{1}\right) u\left(\alpha_{2}\right) \\
+u^{2}\left(\delta_{1}\right)+u^{2}\left(\delta_{2}\right)+u^{2}\left(\beta_{1}\right)+u^{2}\left(\beta_{2}\right)-2 r_{2} u\left(\beta_{1}\right) u\left(\beta_{2}\right) \\
+u^{2}\left(\gamma_{1}\right)+u^{2}\left(\gamma_{2}\right)-2 r_{3} u\left(\gamma_{1}\right) u\left(\gamma_{2}\right)
\end{array}}
$$

Where $u\left(\right.$.) denotes standard uncertainty, $r_{1}, r_{2}$ and $r_{3}$ are the correlation coefficients between $\alpha_{1}$ and $\alpha_{2}$, between $\beta_{1}$ and $\beta_{2}$, between $\gamma_{1}$ and $\gamma_{2}$, respectively.

On the other hand, by using the proposed technique to generate random correlated sampling sequences, uncertainty evaluating procedure based on MCM for arm stretch $\Delta R$ is as follows:

1. Calculate correlation matrix $R_{z}$ based on target correlation coefficients of input quantities $\alpha_{1}, \alpha_{2}, \gamma_{1}, \gamma_{2}$, $\beta_{1}$ and $\beta_{2}$ using (9) and (10).

2. Form the Cholesky factor $\mathrm{A}$ of $R_{z}$, i.e the upper triangular matrix satisfies $R_{z}=A^{\mathrm{T}} A$.

3. Generate a random array $\boldsymbol{Z}$ of standard Gaussian variates with dimension $10 \times M$, where $M$ is the trial number of the Monte Carlo simulation. 10 is the number of input quantities.

4. Generate random sampling sequences $\boldsymbol{X}_{\boldsymbol{i}}$ of input quantities $R_{1}, R_{2}, \delta_{1}, \delta_{2}$ in Table 1 , which can be converted from random array $\boldsymbol{Z}_{i}(i=1,2,3,4$, denotes the $i$ th column of $\boldsymbol{Z}$ ): $x_{i}=\mu_{i}+\sigma_{i} \times z_{i}$, where $\mu_{i}$ and $\sigma_{i}$ denote the mean value and standard deviation of input quantities $R_{1}, R_{2}, \delta_{1}, \delta_{2}$, respectively. 
5. Generate random sampling sequences $\boldsymbol{Y}_{i}$ of standard Gaussian variates with correlation matrix $R_{z}$ using mathematical relationship: $y_{i}=A^{\mathrm{T}} \times z_{i}$, where $i=5,6, \ldots, 10$.

6. Generate random sampling sequences of input quantities $\alpha_{1}, \alpha_{2}, \gamma_{1}, \gamma_{2}, \beta_{1}$ and $\beta_{2}$ according to respective accumulation distribution function using (6) and (7).

7. Calculate $\Delta R$ by selecting a value from the random sequences of input quantities in turn and a sequence of $\Delta R$ can be obtained: $\left\{\Delta R^{1}, \Delta R^{2}, \ldots, \Delta R^{M}\right\}$.

8. Calculate mean value and associated standard uncertainty according to the sequences of $\Delta R$. Sort the values $\left\{\Delta R^{1}, \Delta R^{2}, \ldots, \Delta R^{M}\right\}$ in ascending order, and determine the shortest coverage interval $\left[\Delta R^{(L)} \leq \Delta R^{(H)}\right]$ at coverage probability $p$ where $H-L$ equals to the integer part of $p \cdot M+1 / 2$.

The abovementioned Monte Carlo calculating process was implemented by using MATLAB 2010b, and the Monte Carlo trial times were set to $M=1 \times 10^{6}$, which was found to be sufficiently large in this case. Alternatively, Monte Carlo trial times can be determined by adaptive Monte Carlo method, which will reduce greatly computing times, especially for complicated mathematical model of measurement [24, 25]. Based on the two methods, mean values and associated standard uncertainty calculating results of $\Delta R$ at the rotational angular velocities of $5.24 \mathrm{rad} / \mathrm{s}$ and $31.42 \mathrm{rad} / \mathrm{s}$ are shown in Table 3., in which two significant digits were regarded as meaningful. The empirical frequency distributions based on MCM are illustrated in Fig.6., which provides an approximation to the PDF of $\Delta R$. Coverage intervals at the coverage probability of $95 \%$ from GUM and MCM are indicated by dashed and vertical full lines in Fig.6., respectively.

From Fig.6. and Table 3., we can learn that the proposed method based on MCM and GUM gives estimates and associated standard uncertainty of arm stretch $\Delta R$ at different rotational angular velocities in good agreement. As we know, the mathematical model (12) for measurand $\Delta R$ is linear, so the uncertainty propagation model (15) with the first-order Taylor series expansion is accurate enough for calculating standard uncertainty of $\Delta R$. So it can be employed to illustrate the validity of the proposed method for solving measurement problems with correlated input quantities. We can conclude that the proposed method based on MCM is applicable to the uncertainty evaluation and can cope with the cases where the input quantities of measurand are mutually correlated and of non-Gaussian distribution.

It can also be seen from Table 3. and Fig.6. that the coverage intervals at $95 \%$ by the two methods are approximately equal when two significant digits are regarded as meaningful. That is because the output values are approximately characterized by a Gaussian distribution (Fig.6.), and GUM is expected to perform well in cases of calculating expanded uncertainty. Both the absolute differences of the respective endpoints of the two coverage intervals are smaller than the numerical tolerance. It is shown that GUM as well as MCM is effective for evaluating the measurement uncertainty of arm stretch $\Delta R$ with the above measurement approach.

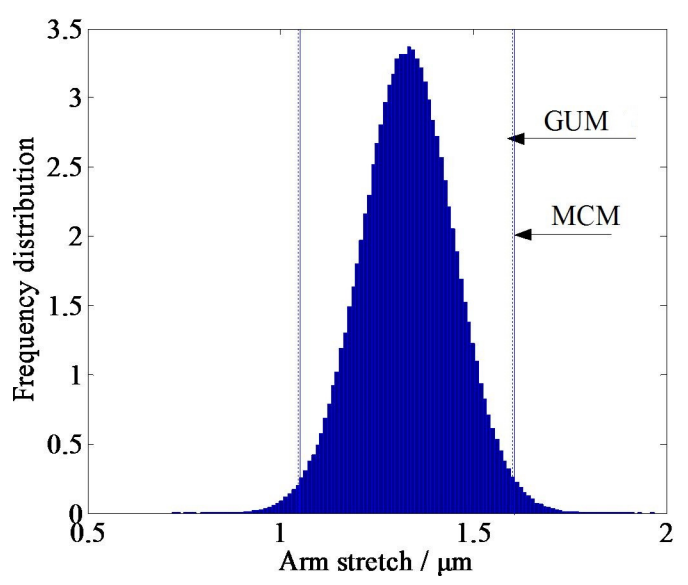

a) Uncertainty calculation result when $\omega=5.24 \mathrm{rad} / \mathrm{s}$.

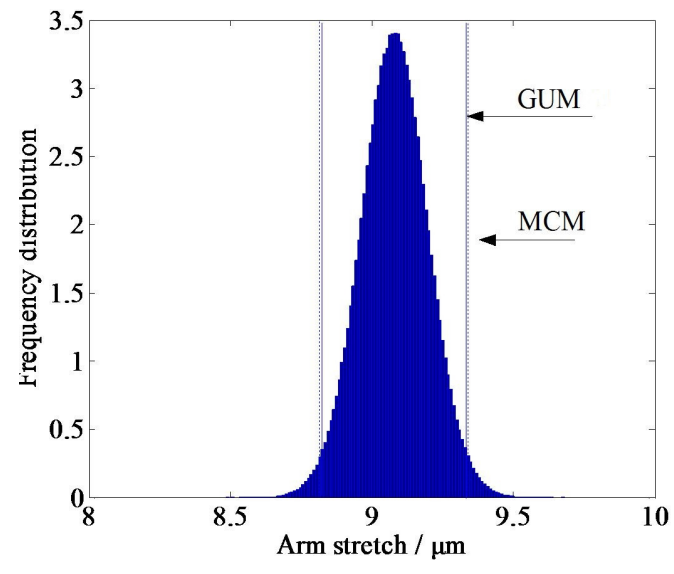

b) Uncertainty calculation result when $\omega=31.42 \mathrm{rad} / \mathrm{s}$.

Fig.6. Empirical PDF of arm stretch based on MCM. Vertical full and dashed lines indicate the (shortest) $95 \%$ coverage intervals determined by GUM and MCM, respectively.

Table 3. Measurement and associated uncertainty results of $\Delta R$.

\begin{tabular}{|c|c|c|c|}
\hline \multicolumn{2}{|c|}{$\boldsymbol{\omega} /(\mathbf{r a d} / \mathbf{s})$} & $\mathbf{5 . 2 4}$ & $\mathbf{3 1 . 4 2}$ \\
\hline \multicolumn{2}{|c|}{$\Delta R / \mu \mathrm{m}$} & 1.36 & 9.11 \\
\hline \multirow{2}{*}{$u(\Delta R) / \mu \mathrm{m}$} & $\mathrm{GUM}$ & 0.121 & 0.119 \\
\cline { 2 - 4 } & $\mathrm{MCM}$ & 0.121 & 0.119 \\
\hline $\begin{array}{c}\text { Coverage } \\
\text { interval at } \\
95 \% / \mu \mathrm{m}\end{array}$ & $\mathrm{GUM}$ & {$[1.093,1.567]$} & {$[8.847,9.313]$} \\
\cline { 2 - 4 } & $\mathrm{MCM}$ & {$[1.094,1.570]$} & {$[8.842,9.313]$} \\
\hline
\end{tabular}

To illustrate the significant contribution of correlation between input quantities to the measurement uncertainty results of measurand $\Delta R$, calculation by using MCM was carried out with the assumption that all input quantities are independent. The measurement results of arm stretch $\Delta R$ and associated coverage intervals at $95 \%$ are shown in Fig.7., the associated standard uncertainty $u(\Delta R)$ at different rotational angular velocities is given in the inset of Fig.7. As can be seen, the standard uncertainty in this hypothetical case is about two times larger than that when some input quantities of $\Delta R$ are considered to be correlated. The reason is that the two input quantities of measurand in mathematical model of measuring $\Delta R$ present a relationship of subtracting, which is conductive to decrease the influence 
of correlated systematical effects on measurement uncertainty of $\Delta R$. It can be concluded that the contribution of correlation between input quantities is significant and cannot be neglected. It is apparent that with the influence of random effects increasing, the difference between the calculation results with full (correlated) model and the standard (uncorrelated) MCM will be reduced.

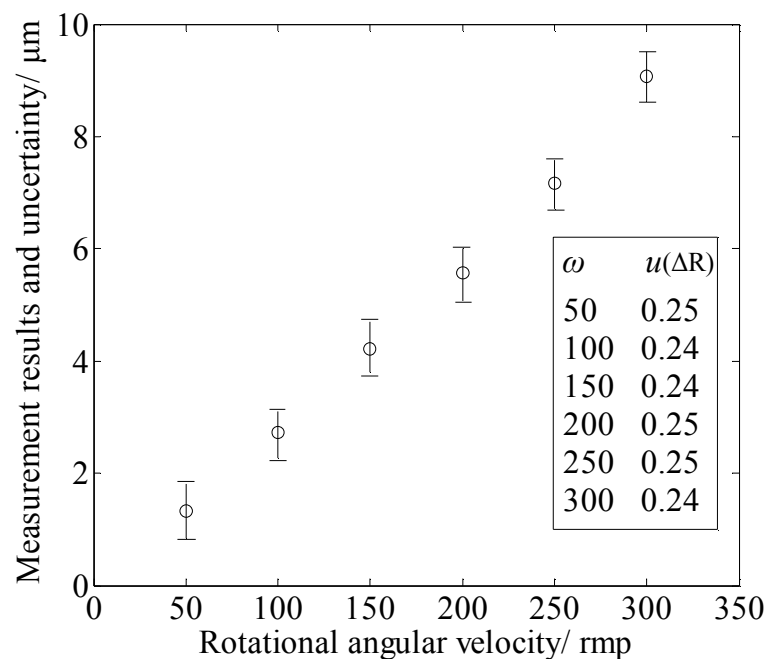

Fig.7. measurement results of $\Delta R$ and associated coverage intervals at $95 \%$ with independent input quantities. The values shown in the inset are the standard uncertainty at different rotational angular velocity $\omega$ of precision centrifuge.

From the above calculation and analysis, it can be concluded that correlated input quantities with a relationship of subtracting or division will weaken the influence of corresponding uncertainty components on the measurement uncertainty of measurand.

\section{CONCLUSION}

In this paper, a general approach for measurement uncertainty evaluation with correlated input quantities is proposed based on MCM, and a linear-nonlinear transforming technique is further developed to generate random sampling sequences of input quantities with target marginal probability distribution and correlation coefficients. The arm stretch of a precision centrifuge with acceleration relative standard uncertainty of $10^{-6}$ order is precisely measured and associated uncertainty evaluation is performed by using GUM and the proposed method. The results show that the two methods give standard uncertainty of the arm stretch $\Delta R$ in good agreement. The proposed method can be a practical alternative to the GUM and MCM in complicated measurement cases with strongly nonlinear measurement model and non-Gaussian distributed nor a scaled and shifted $t$-distributed output, where correlation between input quantities must be taken into account.

\section{ACKNOWLEDGMENT}

The research was supported by the National Key Foundation for Exploring Scientific Instrument of China (No. 2011YQ130047).

\section{REFERENCES}

[1] Joint Committee for Guides in Metrology. (2008). Evaluation of measurement data - Guide to the expression of uncertainty in measurement, 1st edition. JCGM 100:2008.

[2] Martins, M.A.F., Requiao, R., Kalid, R.A. (2011). Generalized expressions of second and third order for the evaluation of standard measurement uncertainty. Measurement, 44, 1526-1530.

[3] Joint Committee for Guides in Metrology. (2008). Evaluation of measurement data - Supplement 1 to the "Guide to the expression of uncertainty in measurement" - Propagation of distributions using Monte Carlo method, 1st edition. JCGM 101:2008.

[4] Eichstad, S., Link, A., Harris, P., et al. (2012). Efficient implementation of a Monte Carlo method for uncertainty evaluation in dynamic measurement. Metrologia, 49, 401-410.

[5] Wen, X., Zhao, Y., Wang, D., Pan, J. (2013). Adaptive Monte Carlo and GUM methods for the evaluation of measurement uncertainty of cylindricity error. Precision Engineering, 37, 856-864.

[6] Theodorou, D., Meligotsidou, L., Karavoltsos, S., et al. (2011). Comparison of ISO-GUM and Monte Carlo methods for evaluation of measurement uncertainty: Application to direct cadmium measurement in water by GFAAS. Talanta, 83, 1568-1574.

[7] Matus, M. (2012). Uncertainty of the variation in length of gauge blocks by mechanical comparison: A worked example. Measurement Science and Technology, 23, 1-6.

[8] Decker, J.E., Eves, B.J., Pekelsky, J.R. (2011). Evaluation of uncertainty in grating pitch measurement by optical diffraction using Monte Carlo methods. Measurement Science and Technology, 22, 1-6.

[9] Moschioni, G., Saggin, B., Tarabini, M., et al. (2013). Use of design of experiments and Monte Carlo method for instruments optimal design. Measurement, 46, 976984.

[10] Chew, G., Walczyk, T. (2012). A Monte Carlo approach for estimating measurement uncertainty using standard spreadsheet software. Analytical and Bioanalytical Chemistry, 402, 2463-2469.

[11] Theodorou, D., Zannikou, Y., Zannikos, F. (2012). Estimation of the standard uncertainty of a calibration curve: Application to sulfur mass concentration determination in fuels. Accreditation and Quality Assurance, 17, 275-281.

[12] Gonzalez, A.G., Herrador, M.A., Asuero, A.G. (2005). Uncertainty evaluation from Monte-Carlo simulations. Accreditation and Quality Assurance, 10, 149-154.

[13] Cox, M.G., Siebert, B.R. (2006). The use of a Monte Carlo method for evaluating uncertainty and expanded uncertainty. Metrologia, 43, S178-188.

[14] Cox, M., Harris, P., Siebert, B.R. (2003). Evaluation of measurement uncertainty based on the propagation of distributions using Monte Carlo simulation. Measurement Techniques, 46, 824-833. 
[15] Herrador, M.A., Gonzalez, A.G. (2004). Evaluation of measurement uncertainty in analytical assays by means of Monte-Carlo simulation. Talanta, 64, 415-422.

[16] Pertile, M., De Cecco, M. (2008). Uncertainty evaluation for complex propagation models by means of the theory of evidence. Measurement Science and Technology, 19, 1-10.

[17] Wubbeler, G., Harris, P.M., Cox, M.G., et al. (2010). A two-stage procedure for determining the number of trials in the application of a Monte Carlo method for uncertainty evaluation. Metrologia, 47, 317-324.

[18] Li, S.T., Hammond, J.L. (1975). Generation of pseudo random numbers with specified univariate distributions and correlation coefficients. IEEE Systems, Man, and Cybernetics, 5, 557-561.

[19] Marida, K.V. (1970). A translation family of bivariate distributions and Frechet's bounds. Sankhya, 32, 119122.

[20] Cairo, M.C., Nelson, B.L. (1997). Modeling and generating random vectors with arbitrary marginal distributions and correlation matrix. Technical Report. Department of Industrial Engineering and Management Science, Northwestern University, Evanston, IL.
[21] Yen, J.C. (2001). NORTA initialization for random vector generation by numerical methods. Masters of Industrial Engineering Thesis. Chung Yaun Chiristian University, Taiwan.

[22] Niaki, S., Abbasi, B. (2008). Generating correlation matrices for normal random vectors in NORTA algorithm using artificial neural networks. Journal of Uncertain Systems, 12 (3), 192-201.

[23] IEEE Standards. (2009). IEEE recommended practice for precision centrifuge testing of linear accelerometers. 836-2009.

[24] Theodorou, D., Zannikou, Y., Anastopoulos, G., et al. (2008). Coverage interval estimation of the measurement of Gross Heat of Combustion of fuel by bomb calorimetry: Comparison of ISO GUM and adaptive Monte Carlo method. Thermochimica Acta, 526, 122-129.

[25] Solaguren-Beascoa Fernandez, M., Alegre Calderon, J.M., Bravo Diez, P.M. (2009). Implementation in MATLAB of the adaptive Monte Carlo method for the evaluation of measurement uncertainties. Accreditation and Quality Assurance, 14, 95-106.

Received May 19, 2014. Accepted October 24, 2014. 